\title{
Spatiotemporal context influences perception of an ambiguous target in visual search
}

\author{
Michael R. Scheessele AND IbRAhim ChaABan \\ Indiana University, South Bend, Indiana
}

\begin{abstract}
The two-stage model of amodal completion or TSM (Sekuler \& Palmer, 1992), and the ambiguity theory (Rauschenberger, Peterson, Mosca, \& Bruno, 2004) provide conflicting accounts of the phenomenon of amodal completion in 2-D images. TSM claims that an initial mosaic (2-D) representation gives way to a later amodally completed (3-D) representation. Furthermore, the 2-D representation is accessible only prior to formation of the 3 -D representation. On the other hand, the ambiguity theory claims that the 2-D and 3-D representations develop in parallel and that preference for one of the coexisting representations over the other may be subject to the influence of spatiotemporal context provided by other elements in the visual display. Our experiments support the claim that, once formed, both representations coexist, with spatiotemporal context potentially determining which representation is perceived.
\end{abstract}

A 2-D image, such as the one shown on the left in Figure 1, offers at least two perceptual interpretations: (1) that of a complete disk partially occluded by a complete square or (2) that of a notched-disk/complete-square mosaic. Given that observers seem to prefer the former interpretation, it invites the question of how a 2-D image ultimately gives rise to the 3-D percept of one object continuing behind another. One theory (Sekuler \& Palmer, 1992), the so-called two-stage model of amodal completion (TSM), suggests that a pictorial occlusion first elicits a mosaic representation, followed by a representation depicting 3-D occlusion (see also Rock, 1983). Once the final representation is formed, the earlier mosaic representation is no longer accessible (in fact, Sekuler \& Palmer conjectured that the mosaic representation may have limited accessibility even prior to formation of the final, amodally completed representation). They also showed that amodal completion occurs within $200-400 \mathrm{msec}$ of stimulus exposure, asserting this as evidence that the visual process underlying amodal completion likely has a "purely perceptual component." (Note that they did not exclude the possibility of other, cognitive components.) Addressing the question of whether an initial mosaic representation could be accessed, Rauschenberger and Yantis (2001) showed, using a visual search task, that a mosaic representation can, indeed, be accessed briefly prior to formation of the final, amodally completed representation.

TSM has been challenged by the more recent ambiguity theory (Rauschenberger, Peterson, Mosca, \& Bruno, 2004). There are two major features of this theory. ${ }^{1}$ The first is the claim that the mosaic representation and the amodally completed representation develop in parallel, with the latter developing more slowly than the former.
The second feature of this theory is the claim that, once formed, either representation is potentially accessible. Furthermore, "extrinsic factors," such as context or set, may determine the representation used as the basis for perception of a stimulus such as the one shown on the left in Figure 1. In a visual search experiment, Rauschenberger et al. (2004) used two types of targets: "separate" and "adjacent." (The top row of Figure 2 shows an example of each.) Separate-target trials provided a baseline for performance in the task, whereas adjacent-target trials facilitated testing between the two theories. Besides target type, other factors were stimulus exposure duration (100 or $250 \mathrm{msec}$ ) and set size (four, six, or eight elements). Exposure durations were selected to differentiate between times at which an amodally completed representation could be assumed to be present $(250 \mathrm{msec})$ and times at which its formation was not yet certain $(100 \mathrm{msec})$. An increasing range of set sizes exposed whether search for a particular target type depended on the nontargets used. A subject was instructed to respond whether a complete disk had been present in a given trial. (In a separate trial, the disk was physically complete; in an adjacent trial, the physically incomplete disk could be perceived as complete but partially occluded by the square.) At $100 \mathrm{msec}$, they found search to be comparably efficient, relative to set size, between adjacent- and separate-target types. Performance, in terms of errors, was much better for separate than for adjacent targets at $100 \mathrm{msec}$, however. ${ }^{2}$ They conjectured that adjacent targets often are not amodally completed by $100 \mathrm{msec}$. At $250 \mathrm{msec}$, they found search to be inefficient for adjacent targets but still efficient for separate targets. (Efficient search for separate targets was always expected because the disk was visibly complete for a separate tar- 


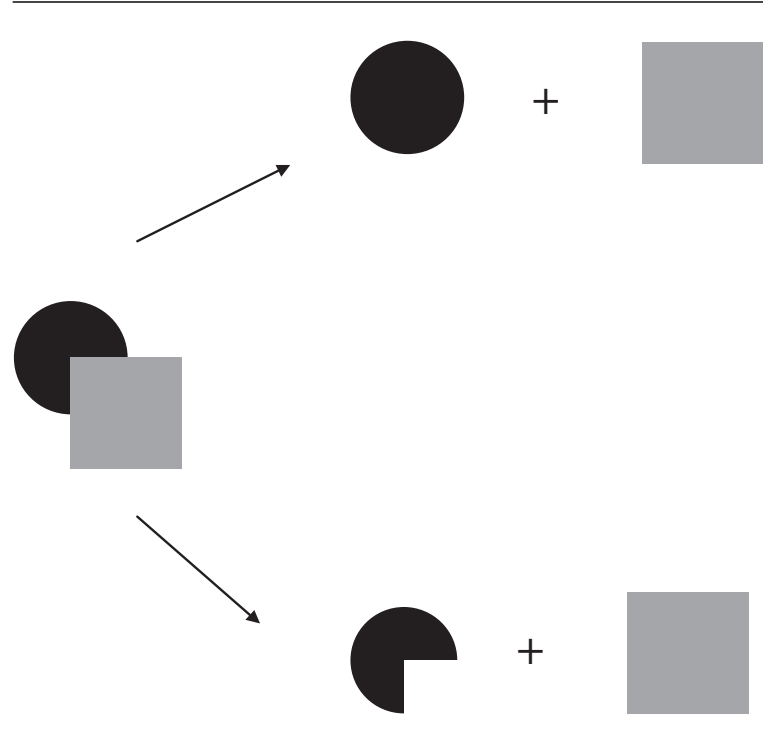

Figure 1. Two possible interpretations for the image on the left.

get, whereas for nontargets the disk was notched.) For an adjacent target detected at $100 \mathrm{msec}$, they concluded that the amodally completed representation had formed and coexisted with the mosaic representation but that the instruction to search for a complete disk "set" subjects to prefer the amodally completed representation. Thus, search was efficient for adjacent targets at $100 \mathrm{msec}$. At $250 \mathrm{msec}$, both representations were assumed present for an adjacent target. They concluded that search was inefficient because the effect of instruction "set" gave way to spatiotemporal context effects of the nontargets. That is, notched disks of nontargets influenced a subject toward the mosaic representation of an adjacent target (Rauschenberger et al., 2004).

However, there is a possible alternative explanation of their results that would neither force abandonment of TSM nor force acceptance of the possibility of spatiotemporal context effects (e.g., the ambiguity theory). Consider Figure 3A, which is similar to various Kanizsa illusions (Kanizsa, 1987). In Figure 3A, an illusory white border can be perceived as surrounding the gray square and partially occluding four complete black disks. Note that an (incomplete) illusory white border can also be perceived as partially occluding the remaining black disk(s) in Figures $3 \mathrm{~B}-3 \mathrm{D}$, although this illusion weakens as disks are removed. Our claim that perception of an illusory white border can occur in the vicinity of the black disks in Figures 3B-3D, even though the "whole" figure in each case does not support the existence of this white border, has prior support. For example, Peterson and Hochberg (1983) demonstrated that observers could respond "to less than the whole stimulus pattern" in cases inspired by the "impossible" figures found in Penrose and Penrose (1958).

Returning to the nontarget in the top row of Figure 2, the alignment of the disk notch with the corner of the square may, therefore, give rise to an incomplete illusory white border in the vicinity of the black disk. Because the illusion is rather weak due to incompleteness of the white border, at $100 \mathrm{msec}$, nontarget disks likely would be perceived as notched disks. At $250 \mathrm{msec}$, however, the illusory white border could be (weakly) perceived to partially occlude a complete disk. Thus, nontargets would be rendered similar to adjacent targets at $250 \mathrm{msec}$ and would cause search to be less efficient, relative to set size. ${ }^{3}$ Experiment 1 tested this possibility.

\section{EXPERIMENT 1}

In the experiment of Rauschenberger et al. (2004), there was inefficiency of search for a disk in adjacent targets in the 250-msec condition. They claimed that this occurred because spatiotemporal context effects provided by nontarget distractors influenced the visual system toward the mosaic representation of an adjacent target stimulus. According to them, search was therefore inefficient because, in effect, an adjacent target was rendered more similar to the nontarget distractors. Our observation, however, is that in their experiment, nontarget distractors may have been rendered more similar to an adjacent target, due to

\section{adjacent separate non- target target target}
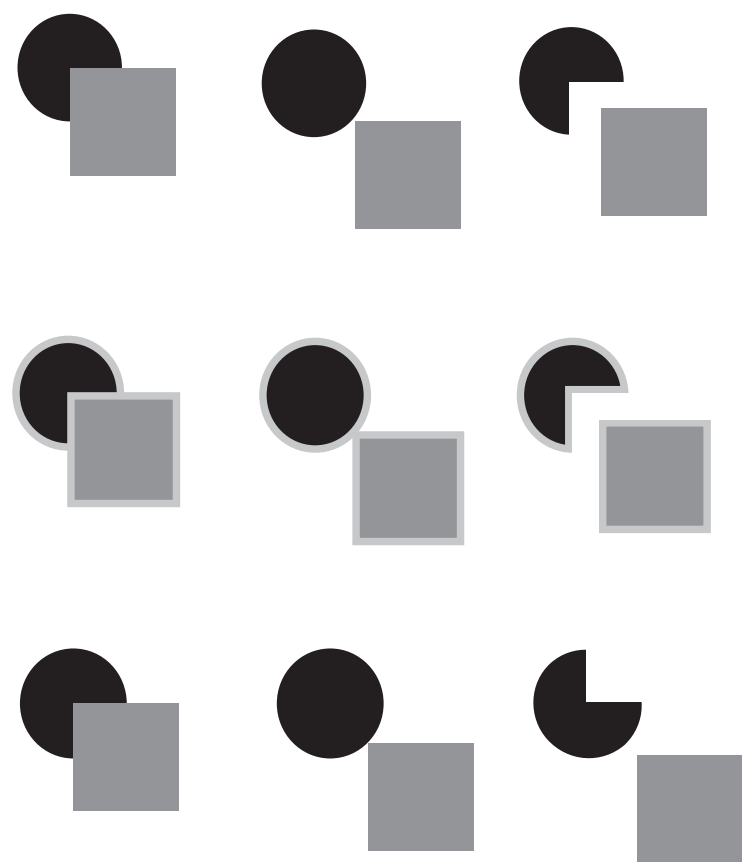

Figure 2. Top row and middle row show types of disk/square pairs used in Experiment 1. Top row and bottom row show types of disk/square pairs used in Experiment 2. (Note that the top row also shows the types of disk/square pairs used in Rauschenberger, Peterson, Mosca, \& Bruno, 2004.) All the geometric elements in the middle row had an explicit red boundary in Experiment 1 (here the boundaries are light gray). In both experiments, the target was a complete disk. In an adjacent target, this was the partially occluded (but presumably complete) disk. In a separate target, it was the complete disk itself. 


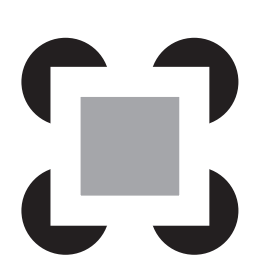

(A)

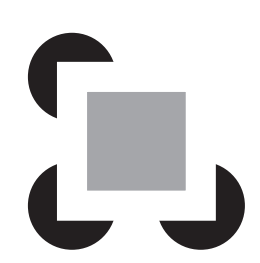

(B)

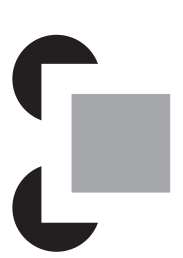

(C)

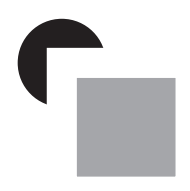

(D)

Figure 3. (A) An illusory white border surrounds the gray square, appearing to partially occlude four complete black disks. (B-D) An (incomplete) illusory white border appears to partially occlude complete black disk(s) in each case, although this illusion progressively weakens with the removal of disks.

process(es) similar to those involved in Kanizsa demonstrations. Such an explanation would account for the inefficiency of search found for adjacent targets in the 250 -msec condition of their experiment. Furthermore, it would not force abandonment of TSM (Sekuler \& Palmer, 1992) in favor of the ambiguity theory (Rauschenberger et al., 2004). In this alternative explanation, the influence of the nontarget distractors on perception of an adjacent target would simply be due to a central, well-known feature of visual search: As the similarity of nontarget distractors to a target increases, inefficiency of search increases. ${ }^{4}$

Performing a better test between TSM and the ambiguity theory required a slight modification of the experiment in Rauschenberger et al. (2004) so that a nontarget distractor would be prevented from forming an illusory white border behind which its notched disk might amodally complete. This modification was implemented by simply adding an explicit boundary to the notched disk and complete square that form a nontarget distractor. Explicit boundaries that enclose a region lead to formation of that region as a unit (Kellman \& Shipley, 1991). Therefore, addition of explicit boundaries to the notched disk and square of a nontarget should disrupt any tendency to perceive a complete disk behind an incomplete illusory white border. In order that a target would not trivially be detected due to lack of an explicit boundary for its component elements, an explicit boundary was also added to the disk and square of both adjacent and separate targets (see Figure 2, middle row). Despite adding explicit boundaries, the ambiguity theory would still predict inefficient search for adjacent targets in the $250-\mathrm{msec}$ condition. TSM, as was pointed out by Rauschenberger et al. (2004), would, on the other hand, predict efficient search for adjacent targets in this condition. The explicit boundaries added here might disrupt the tendency for a nontarget distractor to form an incomplete illusory white border and, in any case, should prevent amodal completion of its notched disk. This manipulation provided a cleaner test between the two theories.

\section{Method}

Subjects. Twenty-five Indiana University South Bend (IUSB) undergraduates participated. All had normal or corrected-to-normal vision. Students who were enrolled in a section of introductory psychology received both course credit and $\$ 15$ for their participation. Students not enrolled in introductory psychology received $\$ 15$.

Stimuli. As in Rauschenberger et al. (2004), targets were complete disks. A target could appear either in an adjacent disk/square pair (notched disk abutting a complete square, where the disk could be seen as complete but partially occluded by the square) or in a separate disk/square pair (a complete disk and complete square). Nontarget distractors were composed of a notched disk positioned near (but not abutting) a complete square. Half of the trials contained a target, and half did not; half of the target trials used an adjacent target, and half used a separate target. For half of the target trials, the disk in a target appeared at the upper left of the square, whereas for the other half, the disk in a target appeared at the lower right of the square. The disk in each nontarget in all the trials appeared either at the upper left or the lower right of the square. The diameter of a disk and the side of a square subtended $1.125^{\circ}$ of visual angle. Each disk/square pair for a trial was placed in one of eight locations along the circumference of an imaginary circle, the center of which was the position of the fixation cross. The diameter of this imaginary circle subtended $8^{\circ}$ of visual angle. A target disk/square pair in a target trial was equally likely to appear in each of the eight locations. In each trial, set size was four, six, or eight disk/square pairs. At most, one of these pairs was a target; all the other pairs were nontarget distractors. Rauschenberger et al. (2004) included trials with just two total disk/square pairs, but as they pointed out, a stimulus display with a set size of two presents subjects with a same-different task, rather than a visual search task. So we did not include a set size of two in our experiment. The only other difference in stimuli between our experiment and theirs was that we used explicit red boundaries for any target/nontarget disk/square pairs in half of the trials. For the other half of the trials, explicit red boundaries were not used. Thus, the no-explicit-boundary trials served as a replication of Rauschenberger et al. (2004). The first two rows of Figure 2 show examples of the types of disk/square pairs used.

Procedure. In Experiment 1, we used a five-factor repeated measures design. The first factor was presence or absence of a target. The second factor was explicit boundary-either present or absent. The third factor was stimulus exposure duration-either 100 or $250 \mathrm{msec}$. The fourth factor was target type (for target trials) - either adjacent or separate. The fifth factor was set size - four, six, or eight disk/square pairs in a stimulus display. There were 16 trials for all possible combinations, giving $2 \times 2 \times 2 \times 2 \times 3 \times 16=768$ total experimental trials, which were divided into 16 mixed blocks of 48 trials each. In addition, before starting the experimental blocks, each subject ran 2 practice blocks of 48 trials each. In the first practice block, stimulus exposure duration was $950 \mathrm{msec}$, rather than 100 or $250 \mathrm{msec}$. The longer stimulus exposure duration was used in the first practice block, in order to aid the subject in learning the task. The subject's task was to report whether a complete disk (target) was present or absent in the stimulus display for a given trial. For target present, the subject was instructed to press the "/" key; for target absent, the " $Z$ " key. Although we measured both accuracy and speed of response, we stressed that we were more interested in accuracy than in speed. The monitor used was approximately 16 in. (viewable) and was positioned $75 \mathrm{~cm}$ in front of a subject. The subject used a chin-and-forehead rest.

On a given trial, a fixation cross appeared for $2 \mathrm{sec}$ on an otherwise blank screen. Then the stimulus display of disk/square pairs appeared for either 100 or $250 \mathrm{msec}$, followed immediately by the mask display, which appeared for $250 \mathrm{msec}$. The mask display consisted of 
colored, randomly positioned lines appearing in the locations where disk/square pairs had just appeared. Finally, a blank screen appeared, and the subject was allowed to respond whether or not a complete disk (target) had appeared. The subject was given feedback, in the form of a beep, for an incorrect response. After the subject's response, the next trial automatically began. In short, the methodology followed that in Rauschenberger et al. (2004), with the exceptions that we did not use a set size of two and we used explicit red boundaries in half of the trials. Given that the nonexplicit boundary trials were intended to replicate their original experiment, we were able to directly examine the effect of adding explicit boundaries.

\section{Results and Discussion}

As in prior studies (Rauschenberger et al., 2004; Rauschenberger \& Yantis, 2001), our analysis focused on target trials only (see Figure 4). Also, as in those studies, our focus was on error data, rather than response time data (see Rauschenberger \& Yantis, 2001, for justification), and, specifically, on the slopes of error functions, relative to set size. However, for completeness, response time data are also provided (see Figure 5). ${ }^{5}$ In Figure 4, solid curves represent our replication of the conditions in Rauschenberger et al. (2004). Dashed curves represent the new conditions in which explicit boundaries were used. As Figure 4 shows, our replication captured the pattern of results found in their study for both the 100-msec (top panel) and the 250-msec (bottom panel) stimulus exposure durations. Furthermore, the new conditions yielded a pattern of results similar to those of the replication conditions, except for an upward translation of the error curve for adjacent targets at both 100 and $250 \mathrm{msec}$.

In the Rauschenberger et al. (2004) study, the key comparisons were the following: relative efficiency of search between adjacent- and separate-target conditions at $100 \mathrm{msec}$, relative efficiency of search between adjacentand separate-target conditions at $250 \mathrm{msec}$, and relative efficiency of search between the 100 and the $250-\mathrm{msec}$ conditions for separate targets. The first comparison served to test for an effect of instruction set (respond target present if complete disk present) on perception of adjacent targets at $100 \mathrm{msec}$. The second comparison served to test for an effect of spatiotemporal context provided by nontarget distractors on perception of adjacent targets at $250 \mathrm{msec}$. Since performance in the separate-target conditions served as a baseline, the third comparison was used to verify that search was comparably efficient (as would be expected) at 100 and $250 \mathrm{msec}$ for separate targets. A three-factor ANOVA was performed on the slopes of the error functions shown in Figure 4. Slope of an error function is an indicator of search efficiency in a visual search task (Rauschenberger \& Yantis, 2001): Increasing slope corresponds to a decrease in search efficiency. The three factors analyzed were explicit boundary (present or absent), stimulus exposure duration (100 or $250 \mathrm{msec}$ ), and target type (adjacent or separate). The stimulus-exposure-duration/target-type interaction was significant $[F(1,24)=7.34, p=.0123]$. The target type main effect was also significant $[F(1,24)=9.21, p=.0057]$. All other interactions and main effects were not significant. In order to analyze the interaction further and to perform the three comparisons found in Rauschenberger et al. (2004), the Scheffé multiple comparison procedure was used. This
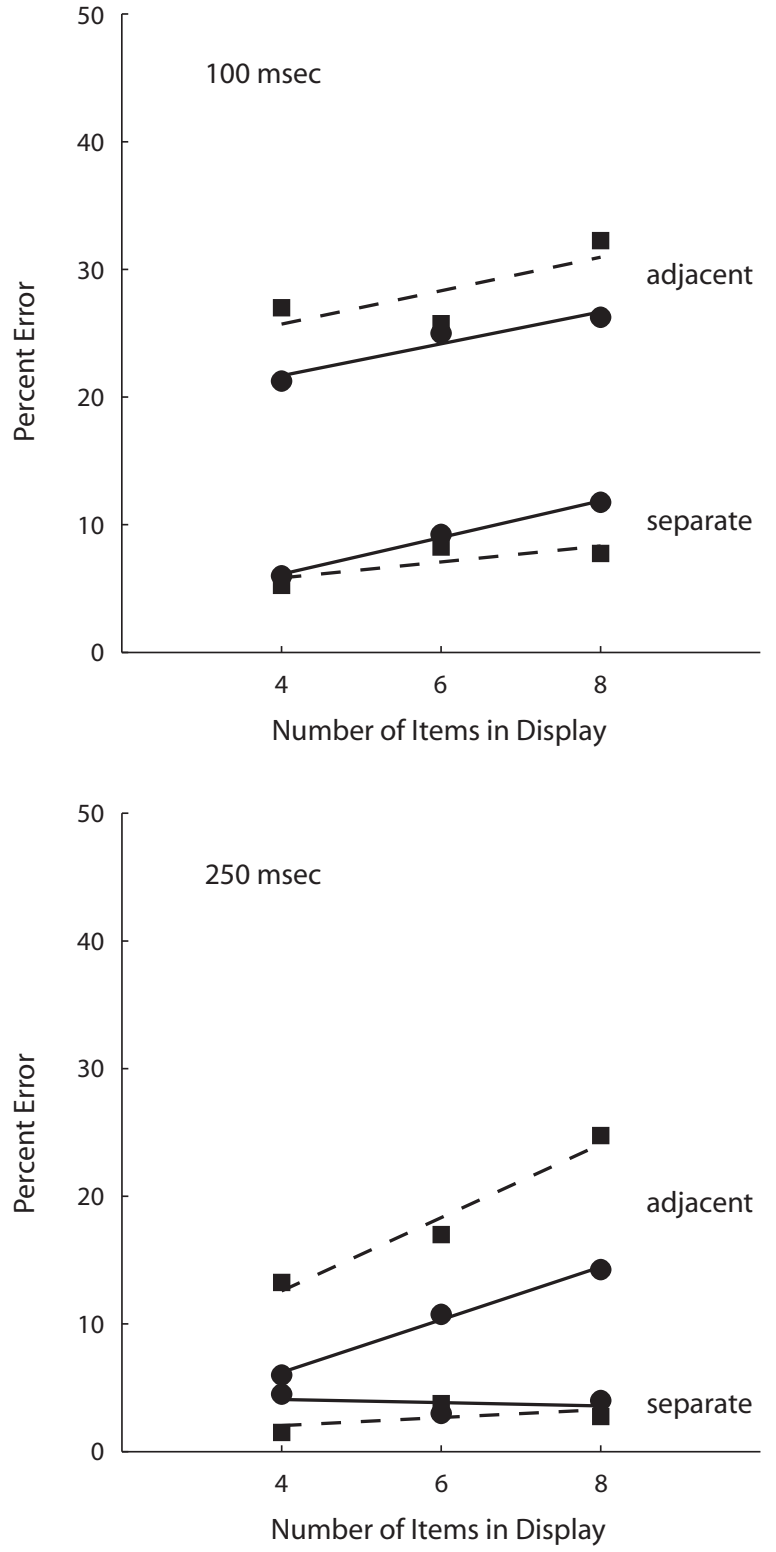

Figure 4. Experiment 1 results (error data). Top panel: 100-msec stimulus exposure duration. Bottom panel: 250-msec stimulus exposure duration. Solid lines represent performance in nonexplicit boundary conditions. Dashed lines represent performance in explicit boundary conditions.

procedure revealed: no significant difference in slope (i.e., comparable search efficiency) between adjacent and separate targets at $100 \mathrm{msec}(p=.9766)$, a significant difference in slope (i.e., inefficient search for adjacent targets, relative to separate targets) between adjacent and separate targets at $250 \mathrm{msec}(p=.0031)$, and no significant difference in slope (i.e., comparable search efficiency) between 100 and $250 \mathrm{msec}$ for separate targets $(p=.4313)$.

Performance in the nonexplicit boundary conditions replicated that reported in Rauschenberger et al. (2004). 

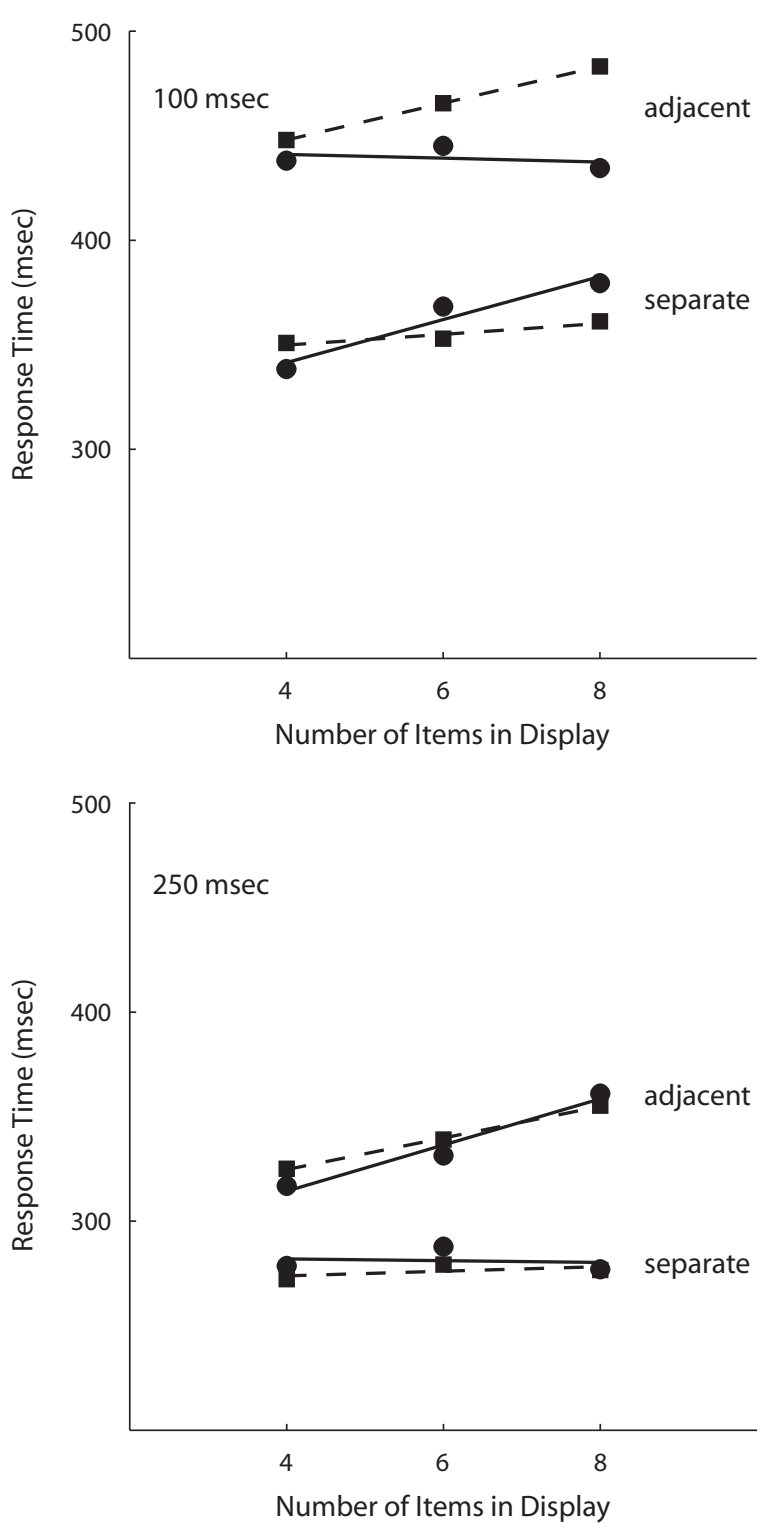

Figure 5. Experiment 1 results (response time data). Top panel: 100-msec stimulus exposure duration. Bottom panel: 250-msec stimulus exposure duration. Solid lines represent performance in nonexplicit boundary conditions. Dashed lines represent performance in explicit boundary conditions.

Adding explicit boundaries did not change this basic pattern of performance. Although phenomenally a nontarget distractor with no explicit boundaries (e.g., right column, top row of Figure 2) may give rise to an incomplete illusory white border for its square element, this apparently does not lead to an amodally complete disk element that interferes with search for the amodally complete disk of an adjacent target. At $250 \mathrm{msec}$, when explicit boundaries were present, search was inefficient for adjacent targets, relative to separate targets. Thus, this experiment supports the ambiguity theory over TSM.
Adding explicit boundaries to disk/square pairs in the stimulus display did have one noticeable effect: It led to more errors in the search for adjacent targets at both 100 and $250 \mathrm{msec}$ (see Figure 4). (An error in the conditions represented by Figure 4 meant that a subject did not report presence of a target that was indeed present.) Recall that, in the Rauschenberger et al. (2004) experiment, there were substantially more errors at 100 -msec stimulus exposure duration in search for adjacent targets, as compared with search for separate targets (note from Figure 4 that we also show this pattern of results). They conjectured that the disk in an adjacent target trial often did not amodally complete by $100 \mathrm{msec}$. Thus, the error rate was higher for adjacent targets than for separate targets (where the disk was always physically complete) at $100 \mathrm{msec}$. Their conjecture may also provide insight into why there were even more errors in the search for adjacent targets at both 100 and $250 \mathrm{msec}$ when explicit boundaries were used in our experiment. Specifically, explicit boundaries added to the notched disk and square of an adjacent target may have hindered the amodal completion of the disk in an adjacent target in many trials. Thus, at $100 \mathrm{msec}$ - and even at $250 \mathrm{msec}$ the representation of a complete disk partially occluded by a complete square may not have formed yet. This conjecture - that an explicit boundary may have inhibited amodal completion of the disk in an adjacent target - is based on evidence from Brown and Koch (1993). They argued that physical closure (e.g., as caused by explicit boundaries) of unoccluded fragments may be a more important factor than presence of the occluder in determining whether the unoccluded fragments lead to perception of the whole partially occluded object (of which the fragments are a part). ${ }^{6}$ A reasonable interpretation of their results, then, is that amodal completion behind an occluder to join unoccluded fragments is inhibited when these fragments have explicit boundaries. Similarly, in Experiment 1, amodal completion of an adjacent target may also have been inhibited by the presence of explicit boundaries.

\section{EXPERIMENT 2}

The results of Experiment 1 support the claim of the ambiguity theory that the mosaic representation and the amodally completed representation of an adjacent target can coexist and that context can bias the visual system to prefer one representation over the other. In particular, if the spatiotemporal context provided by nontarget distractors can influence the visual system to prefer one representation, one would expect this effect to be robust. Experiment 2 tested whether the apparent spatiotemporal context effect provided by nontarget distractors in Experiment 1 is robust. Specifically, if the notched disks of nontarget distractors per se influenced subjects toward the mosaic representation of adjacent targets at $250 \mathrm{msec}$ in Experiment 1, it should not matter whether the notch of the notched disk is aligned or misaligned with the corner of the square in a nontarget distractor. If this does matter, it may indicate that the orientation of the disk notch relative to the corner of the square in nontarget distractors was 
responsible for the bias toward the mosaic representation of adjacent targets at $250 \mathrm{msec}$ in Experiment 1.

\section{Method}

Subjects. Twenty-two IUSB undergraduates participated. (None had participated in Experiment 1.) All had normal or corrected-tonormal vision. Students who were enrolled in a section of introductory psychology received both course credit and a $\$ 15$ gift card (to a bookstore or for IUSB dining services) for their participation. Students not enrolled in introductory psychology received a $\$ 15$ gift card.

Stimuli. In half of the experimental conditions, the stimuli were identical to the nonexplicit boundary stimuli in Experiment 1 (see Figure 2, top row). These conditions replicated those in the main experiment in Rauschenberger et al. (2004). In the other half of the experimental conditions, the stimulus display for each trial contained nontarget distractors with the notch of the disk misaligned with a corner of the square (see Figure 2, bottom row, right column). Explicit boundaries were not used in this experiment. (Note that the replication conditions served as a control for the experiment.)

Procedure. The procedure was identical to that in Experiment 1.

\section{Results and Discussion}

The results for target trials are shown in Figure 6. (For completeness, response time data are also provided in Figure 7.) In Figure 6, solid curves represent our replication of the conditions in Rauschenberger et al. (2004). Dashed curves represent the new conditions, where the notch of the disk was misaligned with a corner of the square for nontarget distractors. The solid curves show that, as in Experiment 1, we replicated the basic pattern of performance found by Rauschenberger et al. (2004). One exception is the adjacent target data point for a set size of four at $100 \mathrm{msec}$. It is not clear why the subjects found this condition much easier here than in their experiment or in our Experiment 1. However, this performance may have been partially due to a speed-accuracy trade-off (see the corresponding data point in the response time graph in Figure 7). Of more interest are the new conditions, which show the same basic pattern of performance as in the replication conditions. The adjacent target curve at $250 \mathrm{msec}$ is translated upward slightly, indicating that misalignment of the disk notches for nontarget distractors somehow caused more adjacent targets to be missed, regardless of set size. It is not likely that the disk of adjacent targets failed to amodally complete by $250 \mathrm{msec}$, because at $100 \mathrm{msec}$, the data points were nearly identical for the aligned and the misaligned conditions for two of the three set sizes. If there was an increased failure of amodal completion of disks in adjacent targets at $250 \mathrm{msec}$, this would also have been expected at $100 \mathrm{msec}$ (recall that this was the case for the explicit boundary conditions in Experiment 1).

A three-factor ANOVA was performed on the slopes of the error functions shown in Figure 6. The three factors analyzed were nontarget distractor disk notch alignment (aligned or misaligned), stimulus exposure duration (100 or $250 \mathrm{msec}$ ), and target type (adjacent or separate). The alignment $\times$ stimulus exposure duration interaction was significant $[F(1,21)=8.7, p=.0077]$. Both the alignment $[F(1,21)=5.17, p=.0336]$ and the target type $[F(1,21)=$ $57.53, p<.0001]$ main effects were significant. All other interactions and main effects were not significant. The interaction effect and alignment main effect can likely
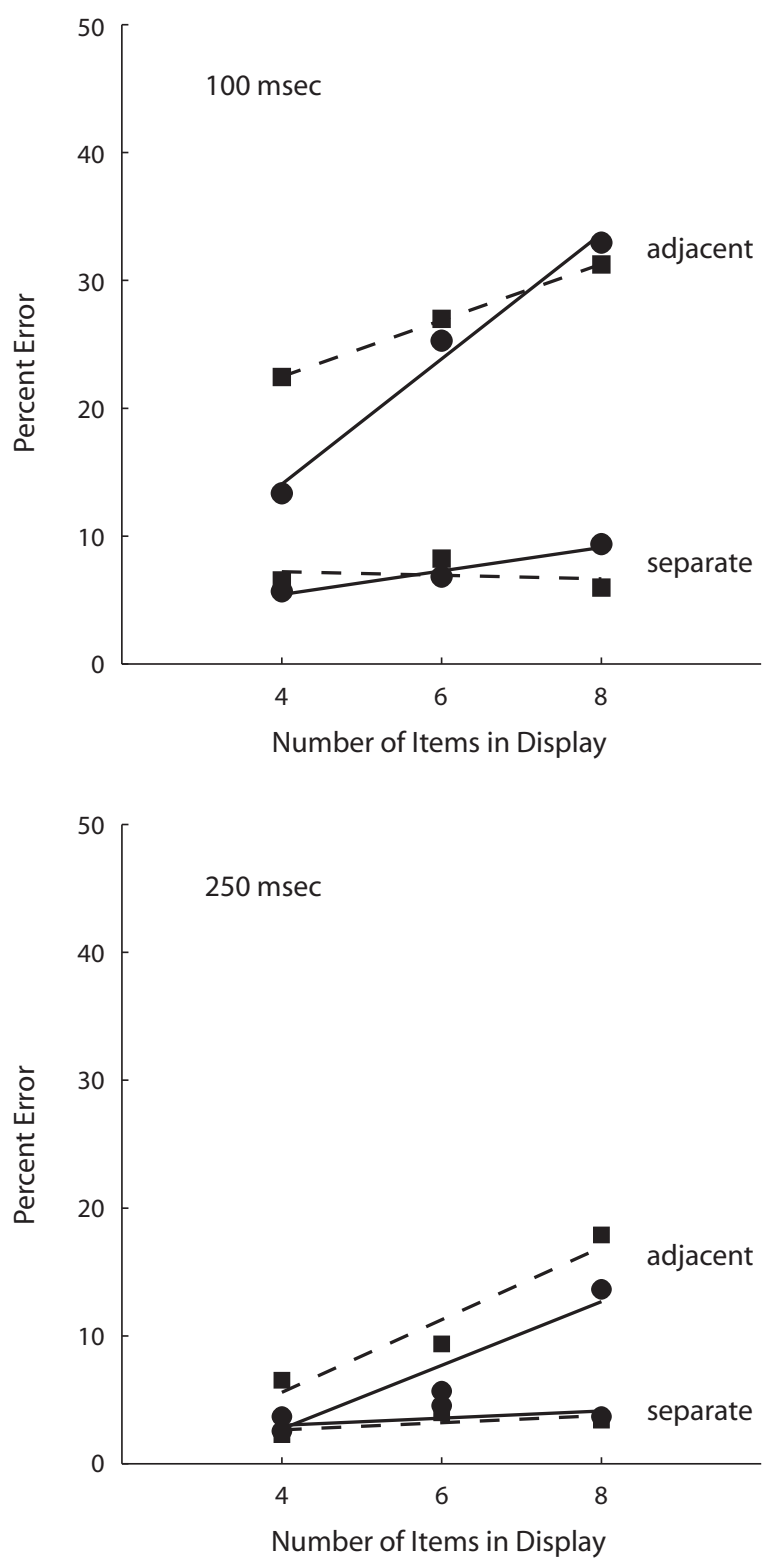

Figure 6. Experiment 2 results (error data). Top panel: 100-msec stimulus exposure duration. Bottom panel: $250-\mathrm{msec}$ stimulus exposure duration. Solid lines represent performance in aligned conditions. Dashed lines represent performance in nonaligned conditions.

be explained by the slope of the adjacent-target/aligneddistractor curve at $100 \mathrm{msec}$. This slope is elevated, due to the data point discussed above. ${ }^{7}$ This elevated slope also likely prevented the stimulus exposure duration $\times$ target type interaction from being significant (it was expected to be significant). This was confirmed by using the Scheffé multiple comparison procedure to perform the three comparisons found in Rauschenberger et al. (2004). This procedure revealed the following: a significant difference in slope (i.e., inefficient search for adjacent targets, relative 

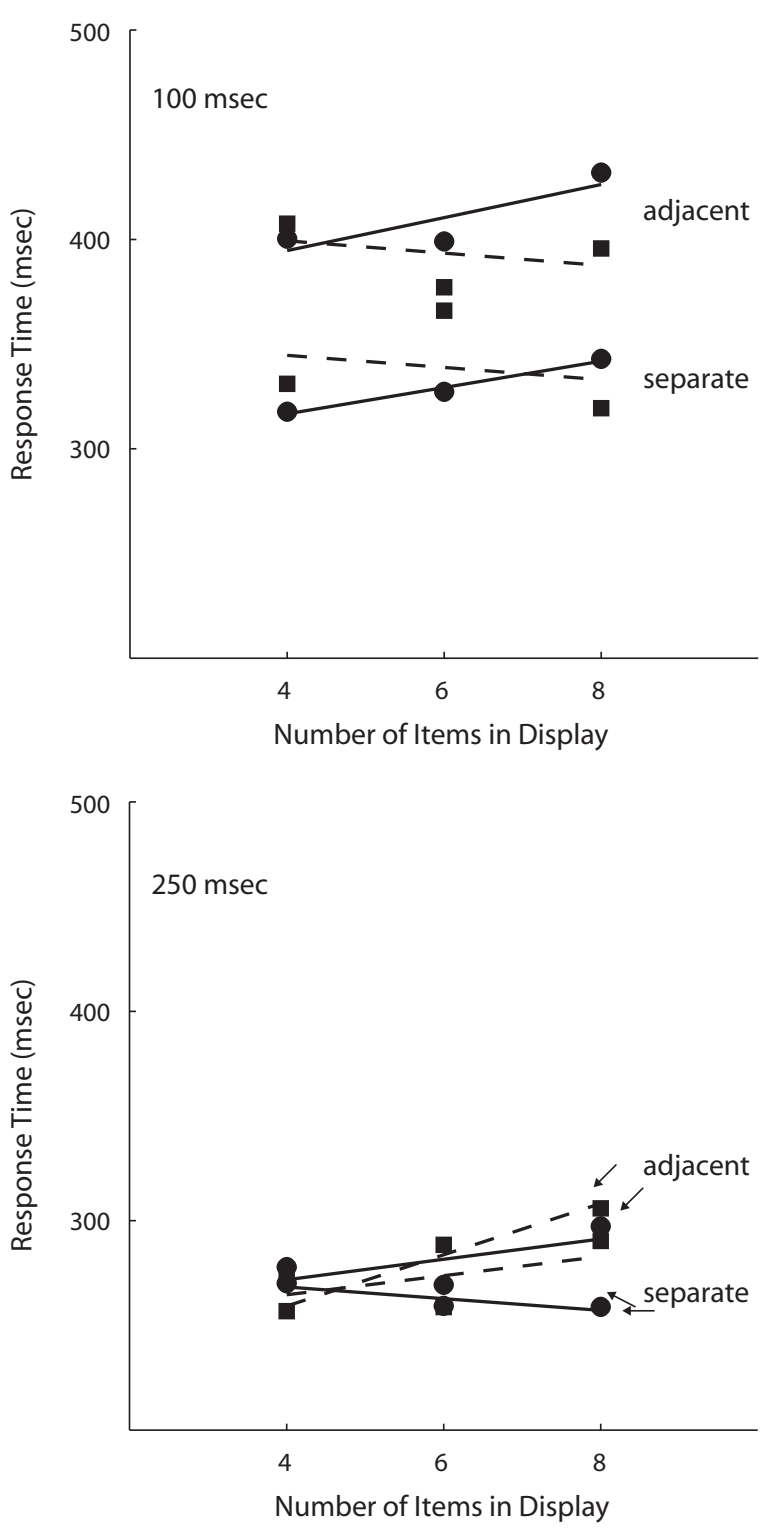

Figure 7. Experiment 2 results (response time data). Top panel: 100-msec stimulus exposure duration. Bottom panel: 250-msec stimulus exposure duration. Solid lines represent performance in aligned conditions. Dashed lines represent performance in nonaligned conditions.

to separate targets) between adjacent and separate targets at $100 \mathrm{msec}(p=.0003)$, likely due to the data point described above; a significant difference in slope (i.e., inefficient search for adjacent targets, relative to separate targets), as was expected, between adjacent and separate targets at $250 \mathrm{msec}(p=.006)$; and no significant difference in slope (i.e., comparable search efficiency), as was expected, between 100 and $250 \mathrm{msec}$ for separate targets $(p=.4313)$. The results of Experiment 2 therefore provide more evidence that spatiotemporal context effects can influence the perception of adjacent targets in visual search; that is, the ambiguity theory is supported. Further- more, however, the effect of nontarget distractors was not caused simply by the orientation of the disk notch relative to a corner of the square; the effect appears to have been due to the notched disk as a whole, without regard to its orientation relative to the square.

\section{GENERAL DISCUSSION}

The results of Experiments 1 and 2 confirm the implication of Rauschenberger et al. (2004) that spatiotemporal context can, in effect, render a target in visual search more similar to nontarget distractors. Naturally, then, this effect would impede the search for a target with increasing set size. (Note that their claim is surprising. It is not simply the well-known fact that as nontarget distractors are made more similar to a target, target search becomes inefficient with respect to set size.) The results of Experiments 1 and 2 demonstrate that this context effect is robust. Furthermore, it is apparent that the mosaic representation and the amodally completed representation can coexist: The mosaic representation does not disappear once the amodally completed representation has formed, as is implied by TSM (Sekuler \& Palmer, 1992). In these two respects, the ambiguity theory is supported over TSM here.

One aspect of ambiguity theory that still requires evidence is the claim that the mosaic representation and the amodally completed representation develop in parallel. By contrast, TSM (Sekuler \& Palmer, 1992) claims that development of the amodally completed representation follows development of the mosaic representation. By now, it is clear that the amodally completed representation generally develops more slowly than the mosaic representation (Rauschenberger et al., 2004; Rauschenberger \& Yantis, 2001; Sekuler \& Palmer, 1992; our results). Also, it is highly probable that both representations, once formed, may be simultaneously present and potentially accessible, depending on context (Gerbino, 1989; Gerbino \& Salmaso, 1987, Experiment 5; Rauschenberger et al., 2004; our results). However, the results thus far do not shed light on the issue of whether the two representations develop in serial or in parallel. Just because formation of the amodally completed representation may take longer than formation of the mosaic representation, it does not logically follow that the former serially follows the latter. Similarly, just because both representations may be accessible, once formed, it does not logically follow that the two representations must develop in parallel. Gerbino doubted that the mosaic representation is a "necessary step" toward the amodally completed representation. However, in his experiments, stimulus exposure durations were used that did not permit testing between the serial and the parallel hypotheses. For example, in Experiment 5 in Gerbino and Salmaso, a stimulus exposure duration of $200 \mathrm{msec}$ was used, and in the experiment reported in Gerbino, a stimulus exposure duration of $350 \mathrm{msec}$ was used. (Recall that Sekuler and Palmer showed that amodal completion could occur by 200 msec.) Bruno, Bertamini, and Domini (1997) found evidence for the "mosaic-first" view when pictorial occlusion was viewed binocularly, but when pictorial occlusion was viewed monocularly or when occlu- 
sion was depicted using binocular parallax, this evidence disappeared. They noted that a pictorial occlusion viewed binocularly provides conflicting depth cues and conjectured that this may correlate with the later presentation of the amodally completed representation in this case. In short, they argued against a mosaic-first view, although they conceded that this possibility could not be ruled out on the basis of their results. Recent neurophysiological evidence (Rauschenberger, Liu, Slotnick, \& Yantis, 2006) indicated that an amodally completed representation evolves from a mosaic representation in the early visual cortex, which subsequently supports both representations. Although this evidence may not require a mosaic stage prior to amodal completion, it seems to hint at this possibility. This suggests a third alternative: The mosaic stage may, indeed, be a required step toward the amodally completed representation, as is suggested by TSM, but the mosaic representation may persist during and after amodal completion, as is specified by the ambiguity theory. Although this alternative is not parsimonious, it is nevertheless logically possible.

Finally, Rauschenberger et al. (2004) introduced another question by suggesting that instruction set caused search to be comparably efficient between adjacent and separate targets at $100 \mathrm{msec}$ (we replicated this result in Experiment 1). By $250 \mathrm{msec}$, they claimed that spatiotemporal context effects superseded an effect due to instruction set. However, their claim of an instruction set effect at $100 \mathrm{msec}$ may be too strong. In those cases in which both representations were available by $100 \mathrm{msec}$, it is possible that there existed a preference for the amodally completed representation independent of instruction set. In fact, Wagemans and d'Ydewalle (1989) demonstrated a slight preference for an amodally completed representation over a mosaic representation in a tunnel effect task. Several investigators (Gerbino, 1989; Peterson \& Hochberg, 1983; Wagemans \& d'Ydewalle, 1989) have shown how a perceptual set can bias an observer toward either a mosaic or an amodally completed representation. Yet an effect of instruction set remains to be shown.

\section{AUTHOR NOTE}

This research was funded by two Indiana University South Bend Faculty Research Grants to the first author. We thank Mary Peterson and Robert Rauschenberger for helpful comments on an earlier draft. We thank David Guthrie and Dean Gottschalk for assistance in performing experiments in a pilot version of this study. We also thank Robert Rauschenberger for providing the experimental software and Yi Cheng for useful comments on data analysis. Correspondence concerning this article should be addressed to M. R. Scheessele, Department of Computer and Information Sciences, Indiana University, South Bend, IN 46634 (e-mail: mscheess@iusb.edu).

\section{REFERENCES}

Brown, J. M., \& Косн, C. (1993). Influences of closure, occlusion, and size on the perception of fragmented pictures. Perception \& Psychophysics, 53, 436-442.

Bruno, N., Bertamini, M., \& Domini, F. (1997). Amodal completion of partly occluded surfaces: Is there a mosaic stage? Journal of Experimental Psychology: Human Perception \& Performance, 23, 1412-1426.

Gerbino, W. (1989). Form categorization and amodal completion: A reply to Wagemans and d'Ydewalle. Acta Psychologica, 72, 295-300.

Gerbino, W., \& Salmaso, D. (1987). The effect of amodal completion on visual matching. Acta Psychologica, 65, 25-46.

Kanizsa, G. (1987). Quasi-perceptual margins in homogeneously stimulated fields (W. Gerbino, Trans.). In S. Petry \& G. E. Meyer (Eds.), The perception of illusory contours (pp. 40-49). New York: Springer. (Reprinted from Rivista di Psicologia, 1955, 49, 7-30)

Kellman, P. J., \& ShiPley, T. F. (1991). A theory of visual interpolation in object perception. Cognitive Psychology, 23, 141-221.

Muise, J. G., LeBlanc, R. S., Blanchard, L. C., \& de Warnaffe, A (1993). Discrimination of the shape of the masked inducing figure precedes perception of the illusory triangle. Perception, 22, 623-628.

Penrose, L., \& Penrose, R. (1958). Impossible objects: A special type of visual illusion. British Journal of Psychology, 49, 31-33.

Peterson, M. A., \& Hochberg, J. (1983). Opposed-set measurement procedure: A quantitative analysis of the role of local cues and intention in form perception. Journal of Experimental Psychology: Human Perception \& Performance, 9, 183-193.

Rauschenberger, R., Liu, T., Slotnick, S. D., \& Yantis, S. (2006). Temporally unfolding neural representation of pictorial occlusion. Psychological Science, 17, 358-364.

Rauschenberger, R., Peterson, M. A., Mosca, F., \& Bruno, N. (2004). Amodal completion in visual search. Psychological Science, 15, 351-355.

RAUSCHENBERGER, R., \& YANTIS, S. (2001). Masking unveils pre-amodal completion representation in visual search. Nature, 410, 369-372.

Rensink, R. A., \& EnNs, J. T. (1998). Early completion of occluded objects. Vision Research, 38, 2489-2505.

Rock, I. (1983). The logic of perception. Cambridge, MA: MIT Press.

Sekuler, A. B., \& Palmer, S. E. (1992). Perception of partly occluded objects: A microgenetic analysis. Journal of Experimental Psychology: General, 121, 95-111.

Wagemans, J., \& D'Ydewalle, G. (1989). The effects of kinetic occlusion and categorization on amodal completion. Acta Psychologica, 72, 281-293

\section{NOTES}

1. Similar ideas were proposed by Gerbino (1989).

2. Recall that error (and response time) curves in visual search have two parameters: slope and intercept. Slope pertains to the concept of efficiency: the steeper the slope, the more inefficient the search. It is possible for two distinct error (or response time) curves to have a similar slope but different intercepts. This happened in Rauschenberger et al. (2004). The separate-target and adjacent-target error curves at $100 \mathrm{msec}$ had comparably shallow slopes (i.e., search was comparably efficient in both cases), but the adjacent-target error curve was translated upward, relative to the separate-target error curve. This reflected more errors in the case of adjacent targets at $100 \mathrm{msec}$, regardless of set size. They conjectured that, at $100 \mathrm{msec}$, amodal completion of an adjacent target had not yet occurred in many trials, thus resulting in the elevated curve. However, for those trials on which amodal completion of an adjacent target had occurred, search was just as efficient as for a separate target.

3. One may object that an incomplete illusory white border (as shown in the right column, top row of Figure 2), although phenomenologically present with unlimited viewing time, would not be perceived with only a 250-msec exposure duration. If so, this would preclude the possibility of amodal completion of a notched disk behind the illusory border at $250 \mathrm{msec}$. However, Muise, LeBlanc, Blanchard, and de Warnaffe (1993) have suggested that illusory shapes may be perceived with as little as an approximately 10 -msec exposure duration. Assuming that illusory surfaces function similarly to "real" surfaces, a 250-msec exposure duration should be sufficient for amodal completion of a notched disk behind the illusory border.

4. At this point, one may object that if the distractors in Rauschenberger et al. (2004) indeed became more like the target at $250 \mathrm{msec}$, why was search for separate targets at $250 \mathrm{msec}$ in Rauschenberger and Yantis (2001) efficient? Recall that in the earlier experiment, distractors were complete-disk/complete-square pairs (e.g., middle column, top row of Figure 2), and separate targets were like the distractors of the later experiment (e.g., right column, top row of Figure 2). Also, the task in Rauschenberger and Yantis was to search for a notched, rather than 
complete, disk. Assuming the possibility that the disk in a separate target was amodally complete at $250 \mathrm{msec}$ behind an incomplete illusory white border, one may argue that search should have been inefficient for this case (just as it was for adjacent targets at $250 \mathrm{msec}$ ). However, search for separate targets at $250 \mathrm{msec}$ in Rauschenberger and Yantis was quite efficient. How can this be explained? First, note that the phenomenon of amodal completion, even with unlimited viewing time, would have been less strong (although possible) for a separate target, as compared with an adjacent target, in Rauschenberger and Yantis. Because "filling-in" of a notched disk may merely be "functional," rather than actual (Rensink \& Enns, 1998), a separate target in Rauschenberger and Yantis may not have been functionally similar enough to the distractors (each of which contained a physically complete disk). By contrast, an adjacent target in Rauschenberger and Yantis may have been functionally more similar to the distractors. In Rauschenberger et al. (2004), on the other hand, where each distractor contained a notched nontouching disk, if such a disk were amodally complete by $250 \mathrm{msec}$, it may have been functionally similar to the amodally completed disk of an adjacent target.

5. In Experiments 1 and 2, outlier response times were eliminated from data presentation (Figures 5 and 7) as follows. First, trials with response times greater than $5 \mathrm{sec}$ were excluded from the computation of the response time mean and standard deviation for each experimental condition. Then, using the response time mean and standard deviation for a condition, each trial in the condition with a response time $\geq 2 S D$ s from the mean or with a response time greater than $5 \mathrm{sec}$ was eliminated. (The distribution of response times for both experiments was positively skewed; no trial had a response time $\leq 2 S D$ s from the mean of the experimental condition to which it belonged.) In Experiment 1 (Figure 5), 321 of 9,600 trials (3.34\%) were excluded as outliers. In Experiment 2 (Figure 7), 251 of 8,448 trials (2.97\%) were excluded as outliers.

6. Amodal completion in their experiment would have resulted in the perceptual "joining" of multiple, unoccluded fragments. In our experiments, amodal completion of the disk in an adjacent target did not join together multiple fragments.

7. As has been mentioned, there is some evidence of a speed-accuracy trade-off for this experimental condition (see Figure 7). Also, note how the response time slope for this case across set sizes (shown in Figure 7) is much shallower than that for the corresponding error curve in Figure 6. In fact, the response time slope is comparable to the error slope for the corresponding conditions (shown in Figure 4) in Experiment 1. (Recall that both Experiments 1 and 2 replicated Rauschenberger et al., 2004, for use as controls.)

(Manuscript received April 13, 2007; revision accepted for publication August 1, 2007.) 